\title{
NON-CONTACT TEMPERATURE MEASUREMENT OF A FALLING DROP
}

\author{
William Hof meister and R.J. Bayuzick \\ Center for the Space Processing of Engineering Materials \\ Vanderbilt University \\ Nashville, TN 37235
}

M.B. Robinson

NASA Marshall Space Flight Center

Huntsville, AL 35812

\begin{abstract}
The 105-meter drop tube at the Marshall Space Flight Center has been used in a number of experiments to determine the effects of containerless, microgravity processing on the undercooling and solidification behavior of metals and alloys. These experiments have been limited. however, because direct temperature measurement of the falling drops has not been available. Undercooling and nucleation temperatures are calculated from thermophysical properties based on droplet cooling models. In most cases these properties are not well known. particularly in the undercooled state. This results in a large amount of uncertainty in the determination of nucleation temperatures. If temperature measurement can be accomplished then the thermal history of the drops could be well documented. This would lead to a better understanding of the thermophysical and thermal radiative properties of undercooled melts. An effort to measure the temperature of a falling drop is under way at Vanderbilt and Marshall Space Flight Center. The technique uses two-color pyrometry and high speed data acquisition. The approach will be presented along with some preliminary data from drop tube experiments. The results from droplet cooling models will be compared with non-contact temperature measurements.
\end{abstract}




\section{INTRODUCTION}

Deep undercooling and the subsequent rapid solidification of metals and alloys leads to possible metastable phase formation and microstructural refinement. For this reason undercooling processes are of great interest to the materials scientists. Containerless processing techniques are used to obtain large undercooling below the equilibrium freezing temperature in liquid metals by eliminating sample/crucible interactions since the crucible is of ten an energetically favorable nucleation site for the solid phase. Drop tubes are used to provide a containerless as well as a microgravity environment for metals processing.

The 105-meter drop tube at Marshall Space Flight Center in Huntsville, Ala., has been used for a number of years to investigate containerless. microgravity processing of refractory metals and alloys [1-6]. A schematic of the Marshall drop tube is shown in Figure 1. The facility is instrumented with an Ircon two-color pyrometer at the top to measure the release temperature of a drop, and a series of silicon photovoltaic detectors are used to record the time after release at which solidification and recalescence occur. From this data the undercooling prior to solidification is calculated. The research reported herein represents the first attempt to measure the drop temperature by non-contact pyrometry and thereby provide an independent check on the undercooling calculations.

The experiments were performed in a $10^{-5}$ torr or better vacuum. Drops of approximately $730 \mathrm{mg}$ were melted from $1.57 \mathrm{~mm}$ diameter MARZ grade niobium wire (Materials Research Corporation) in an electron beam furnace. Details of the experimental technique can be found in references 1,4 , and 6 .

\section{DROP COOLING MODEL}

With knowledge of the drop release temperature and the free fall time to solidification, the temperature at which nucleation occurs and the undercooling of the drop can be calculated. Undercooling is the difference between the equilibrium freezing temperature and the actual solid 
phase nucleation temperature. The radiative heat loss of a sphere is given by [7]:

$$
\mathrm{Q}=-\epsilon \sigma \mathrm{A}\left(\mathrm{T}^{4}-\mathrm{T}_{0}{ }^{4}\right)
$$

where $Q$ is the rate of heat loss, $\epsilon$ is the total hemispherical emissivity, $\sigma$ is the StefanBoltzman constant, $T$ is the temperature of the drop and $T_{0}$ is the ambient temperature. In the cooling calculations $Q$ was calculated as a function of temperature and sample mass for time increments $(\Delta t)$ of $0.001 \mathrm{~s}$; hence, the total heat loss $(\Delta Q)$ for $n$ increments is given by:

$$
\Delta Q=\sum_{q=1}^{n} Q_{q} \Delta t
$$

The heat loss is equal to the change in enthalpy $(\Delta \mathrm{H})$ of the sample so that the temperature at the end of any time increment can be calculated by solving the integral of the liquid heat capacity from the initial temperature $\left(T_{i}\right)$ to the unknown temperature at the time $n$ :

$$
\Delta Q=\Delta H=(m / Z) \int_{T_{i}}^{T} C_{p} d T
$$

where $C_{p}$ is the molar heat capacity of the liquid, $m$ is the sample mass and $Z$ is the atomic number. By assuming a constant heat capacity the expression is easily integrated and solved for temperature:

$$
T=\left(\Delta H Z / \mathrm{mC}_{\mathrm{p}}\right)+\mathrm{T}_{\mathrm{i}}
$$

The assumptions involved in this analysis are that the drop is spherical (since the area is calculated from the mass and liquid density) and isothermal, the heat capacity is constant and there is no radiation exchange between the drop and the walls of the tube. Radiation exchange between the drop and the tube was evaluated according to the method of Incropera and DeWitt [8] and found to be negligible. Deviations from sphericity are negligible in drop tube samples. The Biot number for these samples is on the order of $10^{-2}$ so the thermal gradient is less than $10 \mathrm{~K}$ from the center to the surface. 
The uncertainty in cooling calculations can be assessed using the method of Kline and McClintok [9]. The estimated errors in liquid heat capacity $\left(C_{p}\right)$, the total hemispherical emissivity $(\epsilon)$, and initial release temperature $\left(\mathrm{T}_{\mathrm{i}}\right)$ are found to govern the uncertainty of undercooling calculations. Uncertainty due to the computation of area from mass and liquid density and the uncertainty in the cooling time are small by comparison and can be ignored. The uncertainty in nucleation temperature $\left(\omega_{T}\right)$ can be expressed as:

$$
\omega_{T}=\left[\omega_{T_{i}}^{2}+\left[\left(T_{i}-T\right)\left(\left[L_{\epsilon}^{\omega_{\epsilon}}\right]^{2}+\left[\frac{4 \omega_{T i}}{T i}\right]^{2}\right)^{1 / 2}\right]^{2}+\left[\left(T_{i}-T\right) \frac{\omega_{C}}{C_{p}^{2}}\right]^{1 / 2}\right.
$$

where $\omega$ is the uncertainty of a subscript value. A detailed derivation of this equation can be found in Reference 6.

The total hemispherical emissivity of solid niobium is given as [10]:

$$
\epsilon=-0.144+2.88 \times 10^{-4} \mathrm{~T}-4.46 \times 10^{-8} \mathrm{~T}^{2}
$$

This value was used for the emissivity of the liquid with an assumed uncertainty of \pm 0.03 . The liquid heat capacity of niobium is $40.4 \pm 2 \mathrm{~J} \cdot \mathrm{mol}^{-1} \cdot \mathrm{K}^{-1}$ [11]. An Ircon two-color pyrometer was used to measure the release temperature of the drops to $\pm 30 \mathrm{~K}$. The estimated uncertainty in undercooling calculation from this data is $\pm 50 \mathrm{~K}$.

\section{THEORY OF NON-CONTACT TEMPERATURE MEASUREMENT}

Temperature measurement of a falling drop presents many scientific and technical problems. Small sample sizes (2-8 mm diameter), the acceleration of a free-falling drop, and drop drift away from the tube centerline make tracking of the droplet an extremely difficult task. Reflections from the tube walls add to apparent sample brightness. Consequently, quantitative measurement of radiant flux from the droplet through a narrow bandpass filter is problematic. Because of this, continuous single color pyrometry during free fall is considered 
difficult to implement for reliable drop tube temperature measurements. Some hope, however, can be found in the theory of two-color pyrometry.

The principles of two-color pyrometry have been elucidated by numerous authors [12-14] and the theory will not be derived here. The following simplification of the radiometer measurement equation is taken from G.D. Nutter [15]. For a measurement in a narrow spectral band, $\delta \lambda$, centered about $\lambda$ where the spectral radiance, $\mathrm{L}_{\lambda}$ varies slowly with direction, the signal from a photodetector, $\mathrm{S}$, is:

$$
\mathrm{S}=A_{\mathrm{n}, \mathrm{d}} \Omega_{\mathrm{d}} \mathrm{R}(\lambda) \tau(\lambda) \delta \lambda \mathrm{L}_{\lambda}(\lambda, \mathrm{T})
$$

where $A_{n, d}$ is the projection of the field defining aperture area normal to the optical axis and $\Omega_{\mathrm{d}}$ is the solid angle of radiant flux incident on that aperture. $R(\lambda)$ is the responsivity of the detector and $\tau(\lambda)$ is the transmittance of the optics. The output of an ideal radiometer is then directly proportional to the spectral radiance and the first five terms can be expressed as a constant for a given detector monitoring a given wavelength. Thus:

$$
S_{\lambda}=K(\lambda) L_{\lambda}(\lambda, T)
$$

For a two color pyrometer we have:

$$
\frac{S_{1}}{S_{2}}=\frac{A_{n, d} \Omega_{d} R\left(\lambda_{1}\right) \tau\left(\lambda_{1}\right) \delta \lambda_{1} L_{\lambda}\left(\lambda_{1}, T\right)}{A_{n, d} \Omega \cdot d} \operatorname{R(\lambda _{2})\tau (\lambda _{2})\delta \lambda _{2}L_{\lambda }(\lambda _{2},T)}
$$

If a beam splitter is used such that the aperture and solid angle are equal for both detectors, then the equation can be simplified to:

$$
\frac{S_{1}}{S_{2}}=\frac{K^{\prime}\left(\lambda_{1}\right) L_{\lambda}\left(\lambda_{1}, T\right)}{K^{\prime}\left(\lambda_{2}\right) L_{\lambda}\left(\lambda_{2}, T\right)}
$$

with $K^{\prime}(\lambda)$ given by $R(\lambda) \tau(\lambda) \delta \lambda$. $K^{\prime}\left(\lambda_{1}\right) / K^{\prime}\left(\lambda_{2}\right)$ is therefore the instrument constant and can be 
determined by calibration. From the definition of spectral emissivity, $\epsilon_{\lambda}$, we have:

$$
\frac{S_{1}}{S_{2}}=\frac{K^{\prime}\left(\lambda_{1}\right) \epsilon_{\lambda 1} L_{\lambda b}\left(\lambda_{1}, T\right)}{K^{\prime}\left(\lambda_{2}\right) \epsilon_{\lambda 2} L_{\lambda b}\left(\lambda_{2}, T\right)}
$$

where $L_{\lambda b}(\lambda)$ designates the black body spectral radiance.

The important factor from this discussion is that if a beam splitter is used after the field defining aperture then the ratio of signals is independent of the sample distance. The signal ratio depends only on the spectral radiance ratio and instrument constant. If the ratio of spectral emissivities is known and the instrument calibrated, the equation can be solved for temperature. Since the measurement is independent of distance, it is a great advantage for drop tube temperature measurements, because the technique alleviates the tracking problem.

\section{DROP TUBE PYROMETER}

In order to test the concept of ratio pyrometry in the drop tube a simple instrument was constructed and is shown schematically in Figure 2. Radiant energy from the drop falls on a two inch diameter front surface concave reflector and is reflected onto the end of a one inch diameter fused silica light pipe. The mirror has a $200 \mathrm{~mm}$ focal length and is aluminum coated with a magnesium fluoride overcoat. The light pipe is Suprasil( $R$ ) with flat, polished ends and a circumference polished to 0.05 micron diamond paste. Light exits the pipe to an Inconel coated 50/50 non-polarizing beam splitting cube. The filters are Oriel interference filters centered at $900 \mathrm{~nm}(10 \mathrm{~nm}$ bandwidth at half peak) and $655 \mathrm{~nm}(70 \mathrm{~nm}$ bandwidth). This combination was selected so that the detector signal levels would be similar in the temperature range of interest. EGG HUV-2000B photodiode/op-amp detectors with an active area of $23.4 \mathrm{~mm}^{2}$ and a $200 \mathrm{M} \Omega$ feedback resistor were used. The signal from the detectors was collected sequentially at $10 \mathrm{kHz}$ with a SOLTEC data acquisition system of 12 -bit resolution with a sample and hold time of 250 ns. 


\section{EXPERIMENTAL PROCEDURE}

The instrument was placed in the drop tube at the eleventh level, $32 \mathrm{~m}$ from the furnace. In previous tests niobium was found to undercool consistently to a calculated value, $\Delta T$, of about $480 \mathrm{~K}( \pm 12 \mathrm{~K}$ at one standard deviation, $\pm 50 \mathrm{~K}$ uncertainty). At this level of undercooling the total solidification time is on the order of $0.5 \mathrm{~s}$ for the drop sizes used in this experiment. Therefore, after the initial rapid recalescence the drop will remain at the equilibrium freezing temperature for this period of time, and the signal ratio should remain constant. The instrument was tested in this manner.

\section{EXPERIMENTAL RESULTS}

\subsection{TWO-COLOR RESULTS}

A total of 12 drops was recorded. Figure 3 shows the signal ratio calculation for one of the better experiments. The sharp increase at about $90 \mathrm{~ms}$ is due to recalescence. The mean signal ratio at the melting temperature for all 12 drops was $1.163 \pm 0.05$ (one standard deviation). There was a slight decreasing trend of ratio with distance from the pyrometer. Correcting for this factor the instrument was able to measure the freezing temperature to $\pm 50 \mathrm{~K}$ (one standard deviation).

As can be seen in Figure 3, the ratio decreases sharply at $280 \mathrm{~ms}$ where the drop should maintain a constant temperature until about $600 \mathrm{~ms}$. In addition a consistent dip in the ratio and a rise in both channels at 650 ms past the pyrometer, which corresponds to the position of a flange in the drop tube, was observed. This flange causes the $900 \mathrm{~nm}$ wavelength signal to increase more than the $650 \mathrm{~nm}$ wavelength. It is clear from these results that spectral reflections in the drop tube prevent accurate temperature determination with two-color pyrometry. Therefore, the drop tube must be coated with a non-reflecting surface before the two-color technique can be exploited. More consistent results were found when the data was evaluated using each channel as a single color pyrometer. 


\subsection{SINGLE COLOR DATA EVALUATION}

The radiometer measurement equations $(7 \& 8)$ allow the evaluation of the instrument constant $K(\lambda)$ for observation of an object of known spectral radiance. Since $A_{n . d}$ and $\Omega_{d}$ are different for each drop's position, $K$ and the signal level are now a function of an adjustable geometric parameter $(x)$ and equation 8 must be modified. We now have:

$$
\mathrm{S}_{\mathrm{i}}=\dot{\mathrm{K}}_{\mathrm{i}}\left(\lambda, \mathrm{x}_{\mathrm{i}}\right) \mathrm{L}_{\lambda}(\lambda, \mathrm{T})
$$

where $x_{i}$ corresponds to a particular geometric configuration or drop position. This factor changes with distance and radial position in the tube.

A GE 20A/T24/2 tungsten strip lamp calibrated at NBS in 1980 for radiance temperatures at $655 \mathrm{~nm}$ was used for pyrometer calibration. Fused silica condenser and focusing lenses were used to image the desired portion of the filament on a $1 \mathrm{~mm}$ aperture. The pyrometer assembly including the mirror was calibrated at seven distances from the aperture to the mirror $(0.9$ to 1.9 meters, corresponding to signal levels in the linear range of the detector assembly) and nine spectral radiance temperatures at each distance. This procedure is accurate $( \pm 5 \mathrm{~K})$ for $\lambda=655 \mathrm{~nm}$ since the strip lamp was calibrated for blackbody spectral radiance at that wavelength. To determine the spectral radiance at $\lambda=900 \mathrm{~nm}$ the tungsten emissivity data of DeVos [16] was used with a contributing error of $\pm 15 \mathrm{~K}$.

From the laboratory calibration data it is possible to define seven instrument constants for the seven calibration distances at 655 and $900 \mathrm{~nm}$. Instrument constants for each temperature agreed to better than $0.5 \%$ at a given distance (or geometrical configuration) and wavelength. Since the detector response is linear with incident power, $\mathrm{K}_{\mathrm{i}}$ at constant $\lambda$ and $\mathrm{T}$ vary linearly with signal level and can be described to better than $1 \%$ by a single slope. With knowledge of the radiance melting temperature of pure niobium, $\mathrm{K}_{\mathrm{i}}$ for a particular $\mathrm{x}_{\mathrm{i}}$ can be uniquely defined by the signal level at the melting point of niobium in the drop tube.

Figure 4 shows a typical trace of data from one channel. The time of nucleation is designated as $t_{n}$. Rapid recalescence occurs af ter nucleation and continues for approximately $200-400 \mu \mathrm{s}$. Recalescence can be distinguished from reflections in the tube because of the 
rapid rise time. Reflections occur over tens of milliseconds. At one millisecond after nucleation the drop is close to the meiting temperature and the data from $t_{n}+1$ ms to $t_{n}+2 m s$ was averaged and taken as the signal level at the melting point. This is used to define $\mathrm{K}_{\mathrm{i}}$ for the drop position, $x_{i}$, at this time. The drop is moving at about $25 \mathrm{~m}$ per second and in $2 \mathrm{~ms}$ moves only $5 \mathrm{~cm}$ away from the detector, and its radial position has negligible change. Hence, the geometrical configuration factor $\mathrm{x}_{\mathrm{i}}$ and $\mathrm{K}_{\mathrm{i}}$ are very close to the same just before and just after $t_{n}$. Therefore, the instrument constant can be used to determine the radiance temperature at nucleation. The instrument constants derived from the niobium equilibrium melting point data in the drop tube are a function of free fall time. By plotting $\mathrm{K}_{\mathrm{i}}$ as a function of $t_{n}$ it was found that the correction to nucleation temperature determination due to the $2 \mathrm{~ms}$ of free fall was less than $3 \mathrm{~K}$. In this manner the nucleation radiance temperature at $655 \mathrm{~nm}$ and $900 \mathrm{~nm}$ was measured for each drop.

\subsection{SINGLE COLOR RESULTS}

The data used in calculating the nucleation radiance temperature is listed in Table 1 . It is interesting that the scatter in the nucleation radiance temperature data ( $\pm 4 \mathrm{~K}$, one standard deviation ) is much less than the scatter in the calculated undercooling ( $\pm 12 \mathrm{~K}$, one standard deviation). A plot of the melting and nucleation radiance temperature data is shown in Figure 5.

If the melting temperature of niobium is taken as $2741 \mathrm{~K}$ then the spectral emissivity at $655 \mathrm{~nm}$ and $900 \mathrm{~nm}$ can be calculated at the melting temperature from the melting radiance temperature data [17]. These values are $\epsilon 655=0.355$ and $\epsilon 900=0.321$. If these spectral emissivities are used for the undercooled liquid to calculate the true nucleation temperature from pyrometer measurements we have $2341 \mathrm{~K}$ indicated for $655 \mathrm{~nm}$ and $2329 \mathrm{~K}$ for $900 \mathrm{~nm}$, which correspond to undercoolings of $400 \mathrm{~K}$ and $412 \mathrm{~K}$, respectively. 


\section{DISCUSSION OF RESULTS}

The single color drop tube pyrometry data indicates there is an $80 \mathrm{~K}$ discrepancy between the measured nucleation temperature and the nucleation temperature calculated using the drop cooling model. This difference is greater than the combined uncertainty of either technique, indicating that problems may exist with the drop cooling model. It is significant that the scatter in nucleation temperatures is less with the non-contact measurement. Nucleation. whether homogeneous or heterogeneous, is expected over a range of temperatures depending on the activation energy barrier and pre-exponential term in the nucleation rate expression [18]. Previous drop tube experiments using the drop cooling model have shown the range to be $20-$ $30 \mathrm{~K}$ for pure metals, but the non-contact temperature measurement data from this experiment indicate the spread is much narrower.

\subsection{PYROMETRY MEASUREMENT}

The nucleation radiance temperature measurement at $655 \mathrm{~nm}$ has a high confidence level. The calibration of the $20 \mathrm{~A} / \mathrm{T} 24$ strip lamp at $655 \mathrm{~nm}$ is unfortunately eight years old. The lamp has had 30 hours use in that time and drift of $1^{\circ} \mathrm{C}$ per 10 hours has been reported [19]. The measurement, however, relies on a relative change in radiance, the true reference being the melting temperature of pure niobium. The true temperature of a drop $1-2 \mathrm{~ms}$ after nucleation can be estimated by modeling the heat flow during rapid recalescence of undercooled metallic droplets, which has been done by Levi and Mehrabian [20]. The external heat flow from the drops in this experiment is less than $10^{3} \mathrm{~W} \cdot \mathrm{m}^{-2} \cdot \mathrm{s}^{-1}$ so that recalescence effects are considered independent of the surrounding heat flow. At undercooling levels of $400 \mathrm{~K}$ aproximately $60 \%$ of the drop will solidify in the initial rapid recalescence phase. Using the Levi and Mehrabian models for a single nucleation event and plane front solidification the average drop temperature would be $12-15 \mathrm{~K}$ below the melting temperature after rapid recalescence, and large thermal gradients exist between the solid and remaining liquid. These drop tube samples, however, solidify dendritically with tip velocities around $20 \mathrm{~m} \cdot \mathrm{s}^{-1}[21]$. This 
increases the interfacial area by orders of magnitude over the plane front model and has an effect on temperature distribution similar to Levi and Mehrabian's model for multiple nucleation primarily because of the decreased thermal diffusion distance. These models predict drop temperatures within $2-3 \mathrm{~K}$ of the melting temperature after recalescence. Thus, the true temperature of the drop in the drop tube is very close to the melting temperature after rapid recalescence. Departures from the melting temperature in the drop af ter recalescence will slightiy underestimate the degree of undercooling using single color pyrometry as described.

Given the excellent linearity ( $<1.0 \%$ deviation) of the detector/op-amp combination and the consistent $\mathrm{K}_{\mathrm{i}}$ evaluations at different wavelengths, distances and temperatures (better than $1.0 \%$ ), it is reasonable to assume a $2 \%$ accuracy in $655 \mathrm{~nm}$ spectral radiance measurements at the nucleation temperature. This corresponds to $\pm 5 \mathrm{~K}$ in radiance temperature determination at $655 \mathrm{~nm}$. The $900 \mathrm{~nm}$ radiance temperature determination has greater uncertainty since a secondary source was not available for calibration at this wavelength. Calculation of the true nucleation temperature from the nucleation radiance temperature measurement requires an assumption for spectral emissivity values at the nucleation temperature. This is problematic, since no data exist for the spectral emissivity of undercooled liquid niobium. The change in spectral emissivity from the melting temperature to the nucleation temperature should be small and somewhat lower than the value at the melting temperature. Using the spectral emissivity values at the melting temperature for calculation of the true nucleation temperature will result in a slight overestimation of the undercooling. Considering all the above factors, the measured true undercooling $(\Delta \mathrm{T})$ is:

$$
\Delta \mathrm{T}=400 \mathrm{~K} \pm 15 \mathrm{~K}
$$

With this confidence in the temperature measurement the cooling calculation must be viewed with some skepticism. 


\subsection{DROP COOLING MODEL}

The release temperature accuracy was quoted as $\pm 30 \mathrm{~K}$. We have seen that the scatter in calculated undercooling is three times the scatter in measured undercooling. Since heat capacity and total emissivity estimates in the calculations do not change, the increased scatter is probably due to inaccuracies in release temperature measurement. Thermal gradients within the drop in the furnace limit the ability to determine the starting enthalpy. One known boundary condition is the solid wire-liquid drop interface which must be at the melting temperature. Also, reflections from the tungsten filament may cause overestimation of release temperature. These two facts, however, would cause the drop enthalpy to be overestimated and shift the drop cooling calculation toward lesser values of undercooling. It is clear that the enthalpy of the drop at release should be measured independently by drop calorimetry.

The use of equation 6 for liquid total emissivity is probably an overestimation of emissivity because of the decrease in surface roughness of a liquid drop. The magnitude of this overestimation is not clear. A decrease of $20 \%$ would be required for agreement with measured undercooling. The assumption of constant liquid heat capacity could also be in error [22]. Little data exists for undercooled heat capacities, but some models suggest that liquid heat capacity does increase with undercooling [23]. The quoted uncertainty in the liquid heat capacity was $2.5 \%$. A constant heat capacity $20 \%$ higher than the value used would be necessary for agreement between calculated and measured values. Possibly, a combination of the above difficulties results in the discrepancy between drop cooling calculations and measured values from single color pyrometry.

\section{CONCLUSIONS}

While two-color pyrometry of falling drops appears feasible, the technique cannot be demonstrated because of reflections in the drop tube. This problem is being addressed by the installation of a non-reflecting sleeve in a portion of the drop tube. The nucleation temperature of undercooled niobium can be measured, however, by using single color techniques. This 
measurement has revealed discrepancies between the previously calculated and observed undercooling. These problems cannot be resolved without continuous temperature measurement during the experiment. This may be accomplished by two-color pyrometry in the drop tube if reflections can be eliminated. Continuous temperature measurement of drops cooled by radiation could provide insight into the thermophysical properties of undercooled liquid metals and should be pursued.

\section{ACKNOWLEDGEMENTS}

The authors are grateful for the support of NASA under Grant NAG8-536 and the Center for Space Processing of Engineering Materials at Vanderbilt under NASA Grant NAGW-810. Mr. Tom Rathz of the University of Alabama at Huntsville provided valuable help with the drop tube experiments. The use of preliminary radiance melting temperature measurements by A. Cezairliyan is appreciated. 


\section{Table I}

\section{RESULTS OF SINGLE COLOR DATA EVALUATION FOR TWELVE NIOBIUM DROPS}

Wavelength (nm)

655

900
Radiance Temperature (K) Melting [17]

2427.5

2294.5
Nucleation

2108

1998
Standard Deviation

of Nucleation Temperature

4

4 


\section{REFERENCES}

1. L.L. Lacy, M.B. Robinson, and T. Rathz, J. Crystal Growth 51:47 (1981).

2. N.D. Evans, W.H. Hofmeister, M.B. Robinson, and R.J. Bayuzick, Met. Trans. 17A:973 (1986).

3. W.H. Hof meister, N.D. Evans, R.J. Bayuzick, and M.B. Robinson, Met. Trans. 17A:1421 (1986).

4. W.H. Hofmeister, M.B. Robinson, and R.J. Bayuzick, J. Appl. Phys. $\underline{49}(20): 1342$ (1986).

5. W.H. Hofmeister, M.B. Robinson, and R.J. Bayuzick, Mat Res. Soc. Symp. Proc. 87:149 (1987).

6. W.H. Hofmeister, Ph.D. Dissertation, Vanderbilt University, May 1987

7. M.W. Zemansky, Heat and Thermodynamics, (McGraw-Hill, 5th edition, 1968).

8. F.P. Incropera and D.P. DeWitt, Fundamentals of Heat Transfer, (John Wiley \& Sons, New York, 1985).

9. S.J. Kline and F.A. McClintok, Mech. Eng. (Jan. 1953), p. 3.

10. A. Cezairliyan, J. Res. Nat. Bur. Stand. 75A:565 (1971).

11. D.W. Bonnell, A.J. Valerga, and J.L. Margrave, Abs. Am. Chem. Soc. Mtn., (Washington, D.C., 1971).

12. P.J. Beckwith and K.C.A. Crane, Rev. Sci. Instru. 53:871 (1982).

13. G.M. Foley, M.S. Morse, and A. Cezairliyan, 6th Int. Temp. Symp. (American Institute of Physics, New York, 1982), p. 447.

14. K. Nhuyen and M.C. Branch, Rev. Sci. Instru. 56(9):1780 (1985).

15. G.D. Nutter, ASTM STP 895 (1985).

16. J.C. DeVos, Physica 20:690 (1954).

17. A. Cezairliyan, NBS Preliminary Data (Aug. 1987).

18. V.P. Skripov, Current Topics in Materials Science: Crystal Growth and Materials, Vol. 2, E. Kaldis and H.J. Scheel, eds. (North Holland Publishing Co., 1977), p. 328.

19. W.R. Waters, J.H. Walker and A.T. Hattenburg, NBS Special Publication, 250-7.

20. C.G. Levi and R. Mehrabian, Met. Trans. 11 B:21 (1980).

21. M.B. Robinson, W.H. Hofmeister and R.J. Bayuzick, to be published. 
22. J.L. Margrave, Collogue Int sur l'etude des Transformations a Haute Temperature avdessus de 2000K, Odeillo. France, 1971, (editions du Centre National de la Recherche Scientific, 71, Paris. France, 1971).

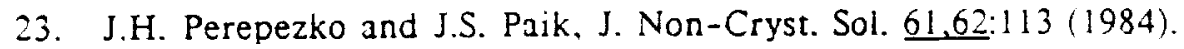




$$
\begin{aligned}
W_{T}=\left\{W_{T_{i}}^{2}+\right. & {\left[\left(T_{i}-T\right)\left(\left[\frac{W_{\varepsilon}}{\varepsilon}\right]^{2}+\left[\frac{4 W_{T_{i}}}{T_{i}}\right]^{2}\right)^{1 / 2}\right]^{2} } \\
+ & {\left.\left[\frac{\left(T_{i}-T\right) W_{c}}{C_{p}}\right]^{2}\right\}^{1 / 2} }
\end{aligned}
$$

FOR CLARIFICATION OF EQ. 5 


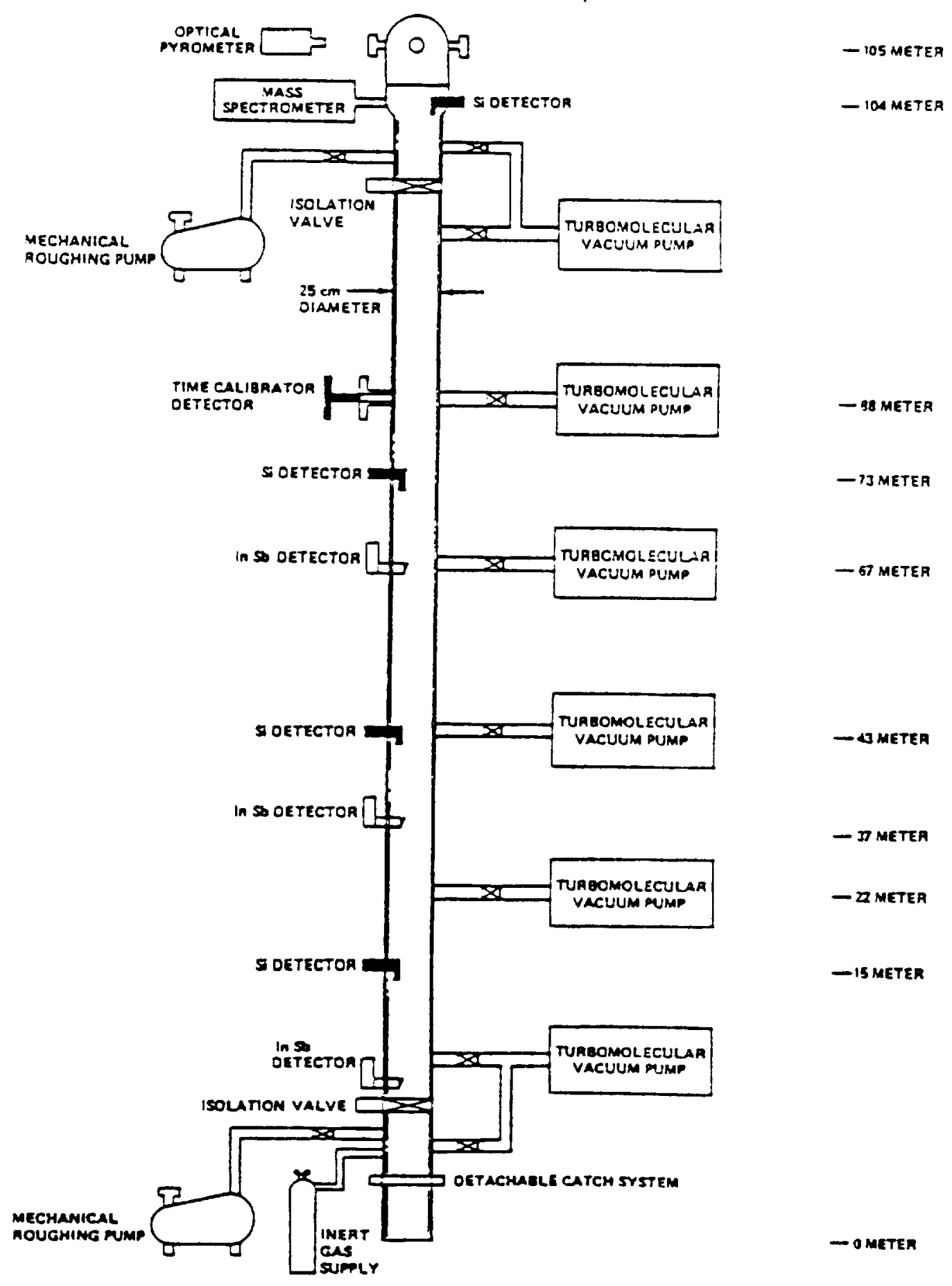

Figure 1. Schematic of the 105-m drop tube at Marshall Space Flight Center. 


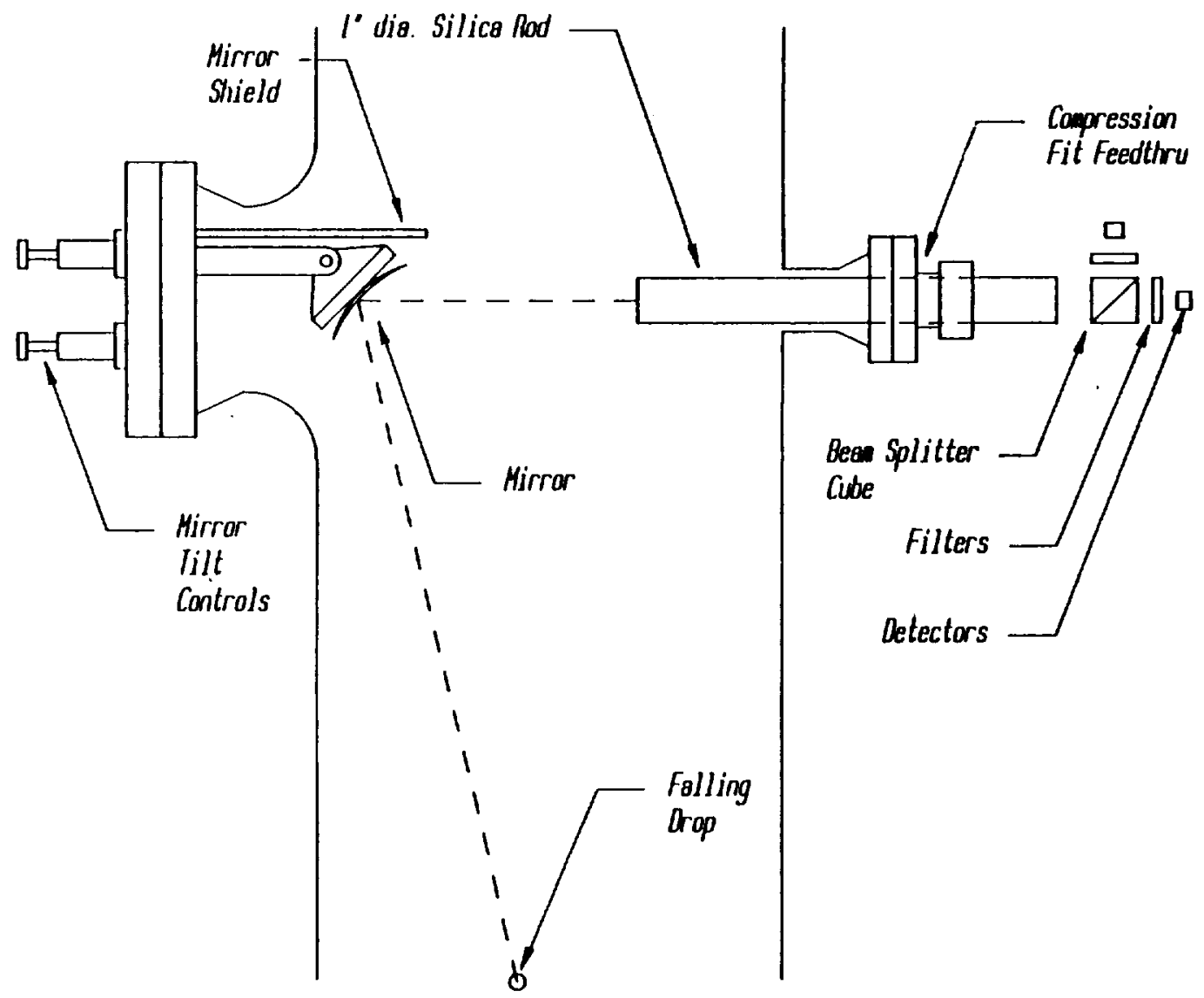

Figure 2. Schematic of the two-color pyrometer as installed in the Marshall Drop Tube.

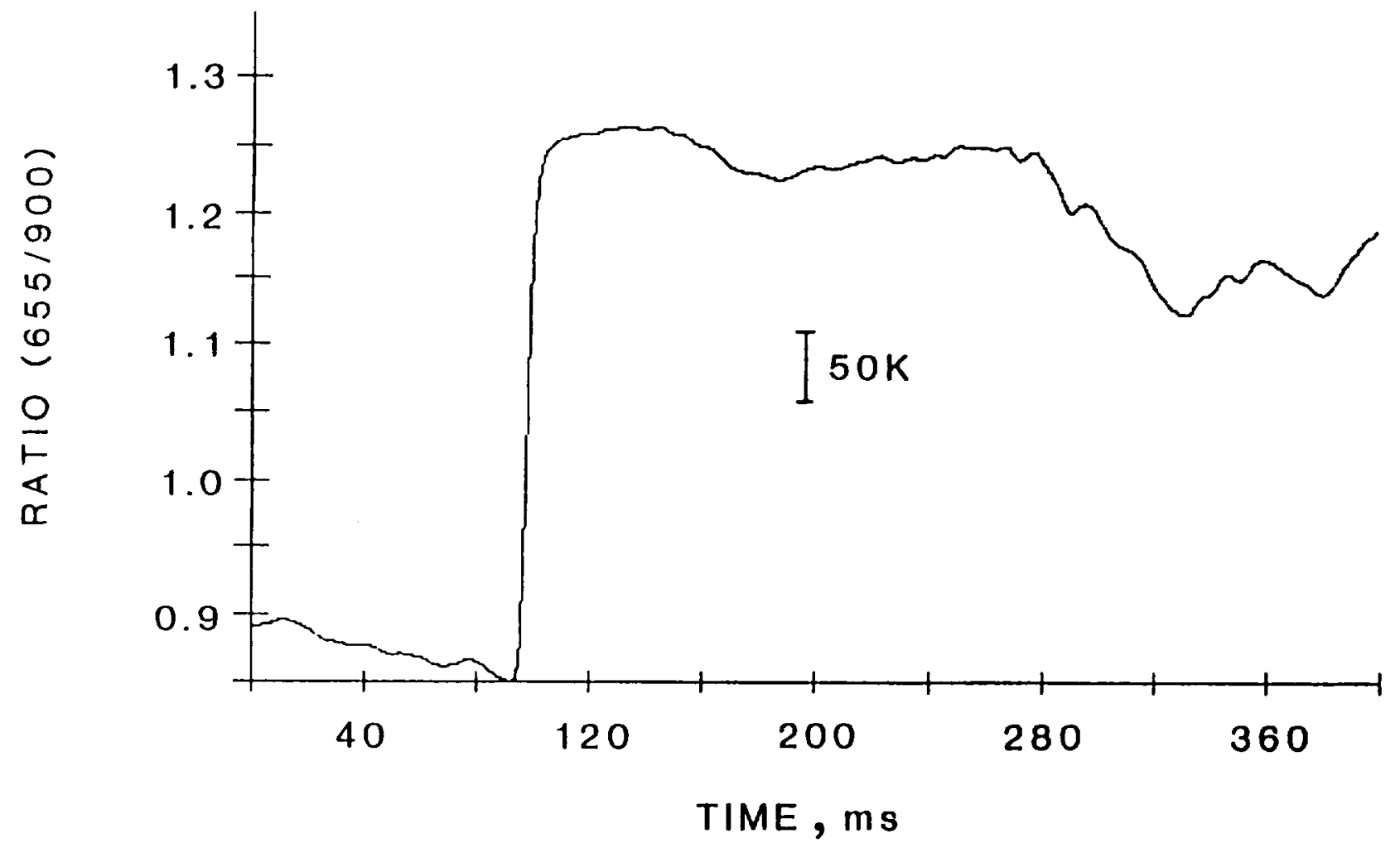

Figure 3. A typical plot of signal ratio data vs, time after passing the mirror assembly. The rise in signal at $100 \mathrm{~ms}$ is due to solidification. 


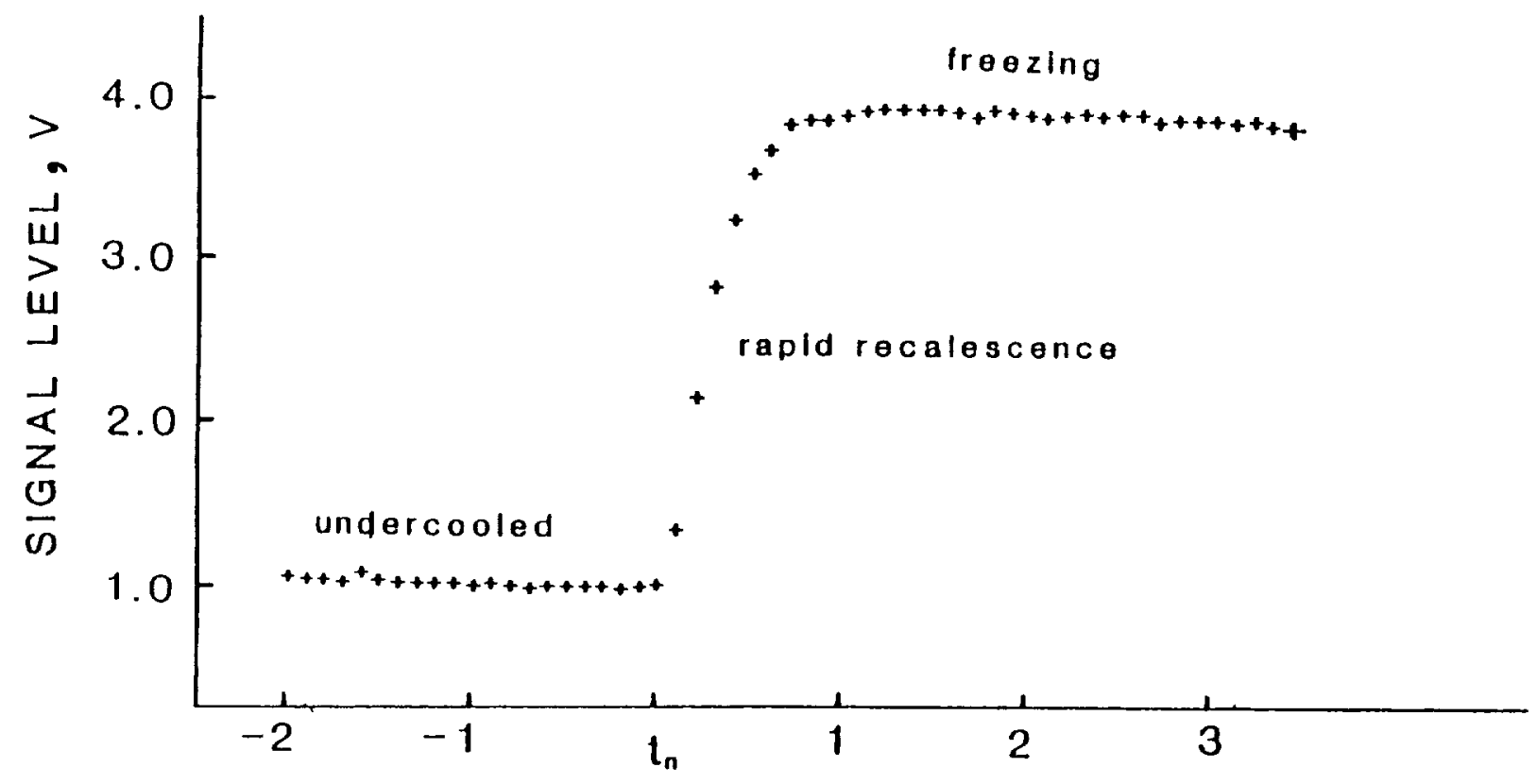

TIME, ms

Figure 4. Single channel data at $655 \mathrm{~nm}$ near the nucieation temperature, $t_{n}$. Data points are taken every $100 \mu \mathrm{s}$.

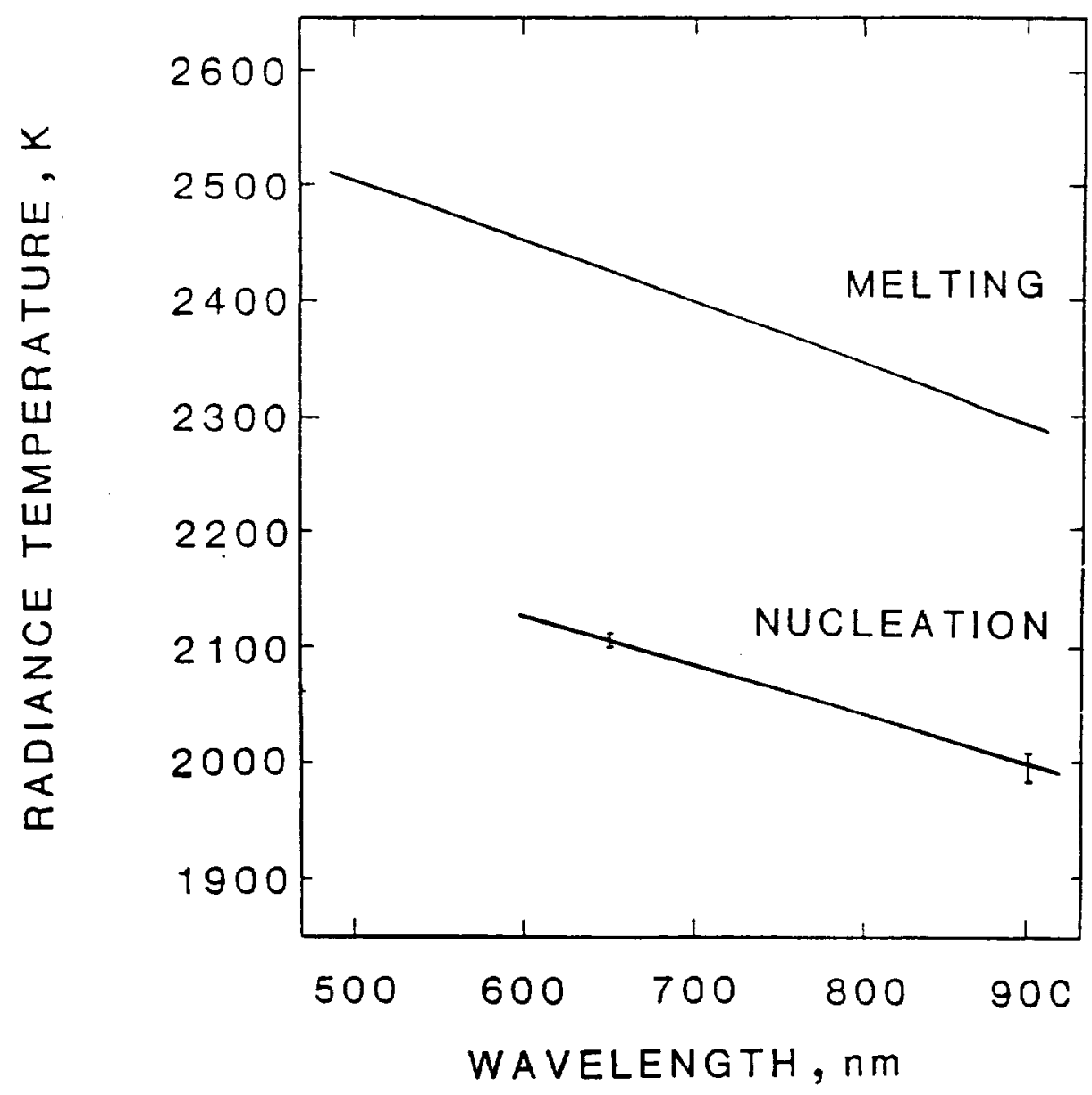

Figure 5. Nucleation radiance temperature as a function of wavelength is plotted with melting radiance temperature data from reference 17 . 